\title{
Toxicological aspects of antineoplastic drugs Doxorubicin and Epirubicin
}

\author{
Zorawar Singh* and Harmanpreet Kaur \\ P.G. Department of Zoology, Khalsa College Amritsar, Punjab, India
}

\begin{abstract}
Doxorubicin (DOXO) and Epirubicin (EPI) are common drugs considerably been used in treating cancer and are sometimes prescribed in combination with other chemotherapeutic drugs. The present review paper elaborates the associated cardiotoxic risk factors related to the administration of both these drugs by going through the available research reports. Patients show signs of irreversible heart damage including arrhythmias, angina, myocardial infarction and cardiomyopathy on treatment with DOXO. It may also result in acute cardiotoxicity in some patients. It has been reported to accumulate in the mitochondria of cardiomyocytes. Similarly, EPI is an analogue of DOXO that has an activity against many forms of cancer. EPI has been used alone or in combination with other agents in the treatment of a variety of malignancies. It is believed to act by intercalating between DNA base pairs and uncoiling the DNA helix, which results in inhibition of DNA synthesis. EPI has proved to be active on a wide spectrum of experimental tumours including L1210, P388 leukaemias and sarcomas. Both DOXO and EPI may significantly upregulate the expression of death receptors including TNFR1, Fas, DR4 and DR5 in induced pluripotent stem cell derived cardiomyocytes at both protein and mRNA levels. Further studies are recommended so as to check the efficacy of both the compounds in the treatment of cancer and associated cardiotoxicities.
\end{abstract}

\section{Introduction}

Doxorubicin (DOXO) is a common drug of class anthracycline and is a hydroxyl derivative of daunorubicin. Adriamycin and rubex are other names for DOXO. It has rubicin as suffix in its name which denotes red color. DOXO has considerably been used in treating cancer and is sometimes prescribed in combination with other chemotherapeutic drugs. DOXO has been found effective in killing of cancer cells for both solid and liquid tumors. It is widely used in the treatment of small cell lung cancer, stomach cancer, breast cancer, ovarian carcinoma, liver cancer, osteogenic bone tumor, thyroid tumor, soft tissue sarcoma, Wilms tumor and neuroblastoma. It is also effective against different types of myelomas, leukaemias and cutaneous T-cell lymphomas [1]. Two different mechanisms, including intercalation of DOXO into DNA and inhibition of topoisomerase II leading to changes in chromatin structure; and generation of free radicals and oxidative damage to biomolecules, have been proposed to explain the mode of action of DOXO in cancer cells [2].

DOXO is usually given to the patient by intravenous injection through central line or a peripheral venous line. DOXO can also be administered by continuous infusion through a central catheter line [3]. The amount of DOXO given to the patient depends upon the weight, height and health status of patient [3]. The duration of treatment depends on the type of cancer, other drugs taken in the treatment and patient's response to the treatment (Figure 1).

\section{Cardiotoxic effects associated with Doxorubicin}

DOXO plays prominent role in treating cancer but its use is limited due to the associated cardiotoxicity risk. Among all the anthracycline drugs, DOXO is known to have a substantial range of associated cardiotoxicity. A serious side effect of DOXO is irreversible heart damage which may include arrhythmias, angina, myocardial infarction, and cardiomyopathy. It may manifest as a reduction in left ventricular<smiles>COc1cccc2c1C(=O)c1c(O)c3c(c(O)c1C2=O)C[C@@](O)(C(=O)CO)C[C@@H]3O[C@H]1C[C@@H](N)[C@H](O)[C@H](C)O1</smiles>

Figure 1. Chemical structure of Doxorubicin

ejection fraction (LVEF) and symptoms of congestive heart failure [4]. Due to this associated cardiotoxicity, a patient can receive DOXO up to certain amount during his lher lifespan. The lifetime maximum dose is $450-550 \mathrm{mg} \backslash \mathrm{m} 2$. It may be lower if patient has some heart disease risk factors such as radiation to the chest, advancing age, and use of other heart-toxic drugs [4]. Within 2-3 days of DOXO administration, it may result in acute cardiotoxicity whereas incidence of chronic cardiotoxicity may occur within 30 days of administration. The incidence of acute cardiotoxicity is $11 \%$ and chronic cardiotoxicity is estimated to be about $1.7 \%$. DOXO associated cardiomyopathy is also influenced by the age of patient. Very young and very old individuals are more prone to develop cardiotoxicity [5]. Treatment with DOXO results in the

*Correspondence to: Zorawar Singh, Department of Zoology, Khalsa College Amritsar, Punjab, India, Tel: +91-9417230075; E-mail: zorawarsinghs@ rediffmail.com

Key words: doxorubicin, epirubicin, cardiotoxicity, malignancy, cancer, cardiomyocytes

Received: December 17, 2019; Accepted: December 26, 2019; Published: December 30, 2019 
increase in mitochondrial DOXO levels whereas cytoplasmic levels of DOXO remain to be low. This clarifies that DOXO accumulates in the mitochondria of cardiomyocytes, which results in a significant increase in mitochondrial iron level and with the treatment of DOXO, oxygen consumption by the mitochondria and mitochondrial membrane get reduced [6] (Table 1).

Autophagy is generally regarded as a protective mechanism that maintains cell viability by recycling unwanted and damaged cellular constituents. In one of the studies, autophagic cell death has been proposed to contribute to DOXO-cardiotoxicity. Additionally, mitophagy, autophagic removal of damaged mitochondria, has been shown to get affected by DOXO in a manner contributing to toxicity [7]. Ultra-violet radiation resistance-associated gene (UVRAG), an autophagy-related protein, is essential for the maintenance of autophagic flux in the heart under physiological conditions. To check the role of UVRAG-mediated autophagy in DOXO-induced cardiotoxicity, mouse models of acute and chronic DOXO-induced cardiotoxicity were used. Deficiency of UVRAG worsen the DOXO-induced mortality and cardiotoxicity was manifested by increased cytoplasmic vacuolization, enhanced collagen accumulation, elevated serum activities of lactate dehydrogenase and myocardial muscle creatine kinase, higher reactive oxygen species (ROS) levels, aggravated apoptosis and more depressed cardiac functions. Experiment with mouse showed that UVRAG deficiency exacerbates DOXO-induced cardiotoxicity through aggravation of DOXOinduced impaired autophagic flux. Intermittent fasting, which restores blunted autophagic flux and ameliorates pathology in the mouse models of DOXO-induced cardiotoxicity, may be used as a potential preventive or therapeutic approach for DOXO associated cardiotoxicity [8].

DOXO and related anthracycline agents including daunorubicin, idarubicin and epirubicin, significantly upregulated the expression of death receptors (TNFR1, Fas, DR4 and DR5) in induced pluripotent stem cells (iPS)-derived cardiomyocytes at both protein and mRNA levels. As a result, iPS- cardiomyocytes underwent spontaneous apoptosis which was further increased by physiologically related death ligands including TNF-related apoptosis inducing ligand, TRAIL. Furthermore, TRAIL potentiated DOXO-induced decrease in beating rate and amplitude of iPS-derived cardiomyocytes. These observations revealed that the induction of death receptors in cardiomyocytes is likely to be a critical mechanism in DOXO related cardiotoxicity [9]. Most models of DOXO cardiotoxicity involve intraperitoneal injection of high-dose drug, which elicits lethargy, anorexia, weight loss, and peritoneal fibrosis, all of which confound the interpretation of autophagy. It was also reported that DOXO blocks cardiomyocyte autophagic flux in-vivo and in cardiomyocytes in culture. This blockage is done by impairing lysosomal acidification and function [10].

\section{Cardiotoxic effects associated with Epirubicin}

Epirubicin (EPI) is the epimer of DOXO, with inversion of the $4^{\prime}$-hydroxyl group on the sugar moiety. It has been used alone or in combination with other agents in the treatment of a variety of malignancies [11]. It is believed to act by intercalating between DNA base pairs and uncoiling the DNA helix, which results in inhibition of DNA synthesis and the normal DNA breaking. These actions may lead to apoptosis of rapidly dividing cells. EPI is an analogue of DOXO that has an activity against many forms of cancer but is used largely in the treatment of advanced breast cancer. EPI has proved to be active on a wide spectrum of experimental tumours including L1210, P388 leukaemias and sarcomas [12]. EPI was approved for use in the United States in 1999. EPI is available as a solution generically in single use vials. The dose of EPI is typically 60 to $100 \mathrm{mg} / \mathrm{m}^{2}$ and is usually given in combination with cyclophosphamide and fluorouracil. Current indications are limited to use in patients with breast cancer who have evidence of lymph node involvement after primary resection of the breast tumor. It is administered by slow intravenous infusion on days 1 and 8 of 28-day cycles. Common side effects are similar to those of DOXO and include cardiac toxicity and secondary malignancies [13]. The mechanism of action of EPI is related to its ability to bind to DNA [12]. Cell culture studies have shown rapid cell penetration, localization in the nucleus and inhibition of nucleic acid synthesis and mitosis [12].

From experimental and clinical studies, it appears that in general, DOXO and EPI exhibit no qualitative, but only some quantitative differences. Thus, the pharmacokinetic and pharmacodynamic characteristics of the two drugs are essentially similar, as are the tumour spectrum and the level of their clinical efficacies. To achieve haematological equitoxicity of the two drugs the dose of EPI should be approximately $20 \%$ higher than that of DOXO, giving rise to a higher cumulative dose of EPI. On the other hand, EPI is significantly less cardiotoxic than DOXO. Thus, the recommended cumulative dose of DOXO is $500 \mathrm{mg} / \mathrm{m}^{2}$ whereas for EPI is $1000 \mathrm{mg} / \mathrm{m}^{2}$ [14] (Figure 2 and Table 2).

To evaluate EPI induced cardiotoxicity occurrence in early breast cancer patients, 6 genes of interest (ABCC1, ABCC2, CYBA, NCF4, RAC2, SLC28A3) and 10 single nucleotide polymorphisms (SNPs)<smiles>COc1cccc2c1C(=O)c1c(O)c3c(c(O)c1C2=O)C[C@@](O)(C(=O)CO)C[C@@H]3OC1C[C@H](N)C(O)[C@@H](C)O1</smiles>

Figure 2. Chemical structure of Epirubicin

Table 1. Studies related to toxicity of Doxorubicin

\begin{tabular}{|c|c|c|l|}
\hline S. No. & Author & Year & \multicolumn{1}{|c|}{ Results } \\
\hline 1 & Koleini et al. & 2017 & $\begin{array}{l}\text { Mitophagy and autophagic removal of damaged mitochondria was affected by DOXO in a } \\
\text { manner contributing to its toxicity }\end{array}$ \\
\hline 2 & An et al. & 2017 & $\begin{array}{l}\text { In acute DOXO induced cardiotoxicity, the survival rate of DOXO treated UVRAG-deficient } \\
\text { mice was significantly lower as compared to DOX-treated WT control mice }\end{array}$ \\
\hline 3 & Zhao et al. & 2017 & $\begin{array}{l}\text { DOXO significantly upregulated the expression of death receptors (TNFR1, Fas, DR4 and } \\
\text { DR5) in iPS-derived cardiomyocytes at both protein and mRNA levels }\end{array}$ \\
\hline 4 & Li et al. & 2016 & $\begin{array}{l}\text { DOXO blocks autophagic flux in cardiomyocytes by impairing lysosome acidification and } \\
\text { lysosomal function }\end{array}$ \\
\hline
\end{tabular}

DOXO, Doxorubicin; UVRAG, Ultraviolet radiation resistance associated gene; WT, Wild type; iPS, induced pluripotent stem cells 
Table 2. Studies related to cardiotoxicity of epirubicin

\begin{tabular}{|c|c|c|l|l|}
\hline Sr. No. & Author & Year & \multicolumn{1}{|c|}{ Results } \\
\hline 1 & Vulsteke et al. & 2015 & $\begin{array}{l}\text { Asymptomatic decrease of LVEF }>10 \% \text { and cardiac failure grade 3-5 (CTCAE 4.0) found after treating the } \\
\text { breast cancer patients with 3-6 cycles of (neo) adjuvant 5-fluorouracil, EPI and cyclophosphamide }\end{array}$ \\
\hline 2 & Feng et al. & 2015 & $\begin{array}{l}\text { DC test was shown to be an effective predictor of the risk of EPI-induced cardiotoxicity } \\
\text { [15] }\end{array}$ \\
\hline 3 & Luo et al. & 2017 & $\begin{array}{l}\text { The total GLS and GLS of the endocardium in every view were significantly reduced in group C (Epi at } \\
\geq 360 \mathrm{mg} / \mathrm{m} 2 \text { ) compared with both groups A (control group) and B (Epi at 180-240 mg/m2) }\end{array}$ \\
\hline 4 & $\begin{array}{l}\text { Framarino-dei- } \\
\text { Malatesta et al. }\end{array}$ & 2015 & $\begin{array}{l}\text { EPI administration in pregnancy should be preceded by the screening of some fetal conditions like SGA and } \\
\text { oligohydramnios that may increase its cardiotoxicity and that, during treatment, the diastolic function of the } \\
\text { fetal right ventricle should be specifically monitored by a pediatric cardiologist }\end{array}$ \\
\hline 6 & Khasraw et al. & 2012 & $\begin{array}{l}\text { The efficacy of EPI is similar to DOXO while EPI has a different toxicity profile particularly for cardiotoxicity } \\
\text { [18] }\end{array}$ \\
\hline 7 & Bi et al. & 2009 & $\begin{array}{l}\text { Two-dimensional strain echocardiography could early and sensitively display the effects of EPI-induced } \\
\text { cardiotoxicity on the systolic function of left ventricular sub-endocardial myocardium, and can early } \\
\text { monitor the EPI-induced cardiotoxicity }\end{array}$ \\
\hline 8 & Okura et al. & 2012 & $\begin{array}{l}\text { EPI-associated cardiotoxicity causes life-threatening heart failure and supportive care is important until the } \\
\text { patient recovers from acute intoxication }\end{array}$ \\
\hline 9 & Stohr et al. & 2006 & $\begin{array}{l}\text { Cardiomyopathy was } 0 \% \text { in patients treated with EPI, and } 0.9 \% \text { in patients treated with DOXO } \\
\text { [21] }\end{array}$ \\
\hline 10 & Nistico et al. & 2007 & $\begin{array}{l}\text { With EPI median dose } 600 \text { mg/m2 and paclitaxel median dose 1760 mg/m2, Troponin-T never overcame the } \\
\text { upper normal limit and also myoglobin never significantly increased }\end{array}$ \\
\hline
\end{tabular}

DC, Deceleration capacity; LVEF, Left ventricular ejection fraction; IP, Intraperitonial; GLS, Gobal systolic longitudinal strain; SGA, Small for gestational age; EPI, Epirubicin

involved in anthracycline induced cardiotoxicity (ACT) were selected. 877 patients treated between 2000 and 2010 with 3-6 cycles of (neo) adjuvant 5-fluorouracil, EPI and cyclophosphamide (FEC) were examined. Main outcome measures were asymptomatic decrease of left ventricular ejection fraction (LVEF) $>10 \%$ and cardiac failure grade $3-5$ (CTCAE 4.0). The impact of clinical factors (age, relative dose intensity of EPI, left-sided radiotherapy, occurrence of febrile neutropenia, and planned and received cycles of EPI) on decrease of LVEF and cardiac failure was also observed. After a median follow-up of 3.62 years (range 0.40-9.60), an LVEF decline of $>10 \%$ occurred in 153 patients $(17.5$ $\%)$ and cardiac failure in 16 patients (1.8\%). No other SNPs related to anthracycline metabolism were retained in the multivariate model for prediction of LVEF decline. ACT in breast cancer patients was found related to number of received cycles of EPI and left-sided radiotherapy [15]. To investigate the clinical value of heart rate deceleration capacity (DC) in predicting the risk of EPI-induced cardiotoxicity, the CK-MB and $\mathrm{cTnI}$ levels and DC values of 86 patients were examined before chemotherapy and after 2 and 4 chemotherapeutic cycles. Patients were divided into low-risk group (LRG) (40 cases), medium-risk group (26 cases), and high-risk group (HRG) (20 cases) based on the calculated DC values. After 4 cycles of chemotherapy, HRG showed a significantly greater increase in serum CK-MB (17.1 \pm 4.9 vs. $14.6 \pm 3.7)$ and $\mathrm{cTnI}$ $(1.28 \pm 0.38$ vs. $1.0 \pm 0.29)$ concentrations over the pre-chemotherapy levels when compared with LRG. After 2 and 4 cycles of chemotherapy, HRG exhibited a significantly greater increase in mean heart rate $(2$ cycles: $79.6 \pm 6.0$ vs. $77.6 \pm 6.7 ; 4$ cycles: $88.2 \pm 10.2$ vs. $82.4 \pm 6.2)$ and the supraventricular ( 2 cycles: $68.9 \pm 19.3$ vs. $57.2 \pm 17.6 ; 4$ cycles: 131.1 \pm 29.5 vs. $91.7 \pm 16.5)$ and ventricular arrhythmia counts ( 2 cycles: $179.0 \pm 20.5$ vs. $162.3 \pm 16.3$; 4 cycles: $228.6 \pm 44.8$ vs. $187.4 \pm 22.6$ ) over the pre-chemotherapy values compared with LRG. DC test was shown to be an effective predictor of the risk of EPI-induced cardiotoxicity [16-23].

Continuous infusion of DOXO has been a strategy to reduce cardiotoxicity. However, evidence is lacking regarding whether this strategy can reduce cardiotoxicity of EPI without compromising antineoplastic efficacy. To check this strategy, EPI healthy rats were randomized into groups: EPI $(8 \mathrm{mg} / \mathrm{kg})$ delivered intraperitoneally via micro osmotic pumps (MOP), EPI ( $8 \mathrm{mg} / \mathrm{kg}$ ) by intraperitoneal (IP) bolus injection, and placebo control. Blood samples were collected for analyzing biomarkers of myocardial injury and pharmacokinetics. Blood biomarkers showed that the degree of myocardial injury in MOP group was lower than that of IP group. Histopathology showed that there was less eosinophilic enhancement, interstitial hemorrhage and necrotizing muscle atrophy in MOP group than IP group. Antineoplastic effect of EPI by MOP was not different from that by IP as measured by tumor weights. This study concluded that slow delivery of EPI by MOP reduced cardiotoxicity without compromising the antineoplastic effect compared to IP bolus delivery [24].

To evaluate the longitudinal and circumferential systolic function of the left ventricular for the early detection of cardiotoxicity, 130 female patients with postoperative breast cancer who did not receive radiotherapy were classified into three groups: Group A (control group, $\mathrm{n}=40)$ without any chemotherapy; Group B $(\mathrm{n}=44)$ administered EPI at $180-240 \mathrm{mg} / \mathrm{m}^{2}$; and Group C $(\mathrm{n}=46)$ administered EPI at $\geq 360$ $\mathrm{mg} / \mathrm{m}^{2}$. Peak and global systolic longitudinal strains (GLS) in the total and endocardium, mid-myocardium, and epicardium were measured and calculated from apical four-chamber, apical two-chamber, and left ventricular long-axis views, respectively. The total GLS and GLS of the endocardium in every view were significantly reduced in group C compared with both groups A and B $(\mathrm{p}<0.05)$, but there was no significant difference between groups $A$ and $B(p>0.05)$. The GLS of the epicardium and mid-myocardium in groups $\mathrm{B}$ and $\mathrm{C}$ were not significantly reduced $(p>0.05)$ [17]. A 38-year-old caucasic woman with a dichorionic twin pregnancy was found at 20 and 1/7 weeks for a suspected breast cancer, later confirmed by the histopathology report. At 31 and 3/7 weeks, after the second chemotherapy cycle, ultrasound examination evidenced the demise of one twin while cardiac examination revealed a monophasic diastolic ventricular filling, i.e. a diastolic dysfunction of the surviving fetus who was delivered the following day due to the occurrence of grade II placental abruption. The role of EPI cardiotoxicity in the death of the first twin was supported by post-mortem cardiac and placental examination. The occurrence of EPI cardiotoxicity in the surviving newborn was confirmed by the report of high levels of troponin and transient left ventricular septal hypokinesia [18]. EPI is an epimer of DOXO with important role in the chemotherapy. The efficacy of EPI is similar to DOXO while EPI has a different toxicity profile particularly in regard to cardiotoxicity [19]. The value of two-dimensional strain echocardiography for assessing left ventricular regional systolic function in breast cancer patients who were treated with EPI was evaluated. A total of 116 breast cancer patients were divided into 3 groups: 38 patients in group A (EPI 120 $340 \mathrm{mg} / \mathrm{m}^{2}, 42$ patients in group B $\left(\mathrm{EPI}>\right.$ or $\left.=360 \mathrm{mg} / \mathrm{m}^{2}\right)$, and 36 
patients after surging without chemotherapy served as the control group C. Two-dimensional strain echocardiography revealed that the peak systolic strain of left ventricular sub-endocardial myocardium in group A was reduced in some segments as compared to controls ( $\mathrm{p}$ $<0.05$ ). It was concluded that $2 \mathrm{D}$ strain echocardiography could early and sensitively display the effects of EPI-induced cardiotoxicity on the systolic function of left ventricular sub-endocardial myocardium, and early monitor the EPI-induced cardiotoxicity [20].

Acute heart failure after chemotherapy with EPI for breast cancer was found in a 42-year-old Japanese woman. Echocardiography revealed a cardiac dysfunction with left ventricular thrombus. Serial serum Troponin-T tests were positive over a 5-week period, and an endomyocardial biopsy demonstrated ultrastructural lesions which were similar to those caused by cardiotoxicities due to DOXO. This case report demonstrated that EPI-associated cardiotoxicity causes life-threatening heart failure and supportive care is important until the patient recovers from acute intoxication [21]. To investigate EPI in children and adolescents, in comparison with DOXO, 172 soft tissue sarcoma patients treated with EPI (median cumulative dose: $450 \mathrm{mg} /$ $\mathrm{m}^{2}$ ) or DOXO (median cumulative dose: $240 \mathrm{mg} / \mathrm{m}^{2}$ ) were examined. Heart function was analyzed by echocardiography and measuring leftventricular fractional shortening. Incidence of clinically manifested cardiomyopathy was found to be $0 \%(0 / 60 ; 95 \%$ CI: $0-6.0 \%)$ in patients treated with EPI, and $0.9 \%(1 / 108 ; 95 \%$ CI: $0-5.1 \%)$ in patients treated with DOXO thus presenting low cardiotoxicity [22]. In another study, 20 patients with untreated advanced breast cancer received EPI $(25 \mathrm{mg} /$ $\mathrm{m}^{2} /$ week) and paclitaxel $\left(80 \mathrm{mg} / \mathrm{m}^{2} /\right.$ week $)$ for 24 weeks. Troponin-T, myoglobin and biochemical serum enzymes circulating levels were measured immediately before and $4 \mathrm{~h}$ after EPI administration every week. Troponin-T never overcame the upper normal limit; one patient experienced Troponin-T elevation without any clinical or instrumental sign of cardiac failure. Myoglobin never significantly increased with the exception of a patient who underwent several abdominal fluid drainages. Creatine kinase MB and C-reactive protein never moved outside the upper normal limit. No symptomatic cardiac event was recorded [23]. Thus, further studies are recommended so as to consider the use of EPI as safe without associated cardiotoxicity.

\section{Conclusion}

Treatment of cancer may involve a single drug or a combination. Doxorubicin (DOXO) and Epirubicin (EPI) are common drugs used in treating cancer. After going through available research papers, it was revealed that patients may show signs of cardiotoxicity including arrhythmias, angina, myocardial infarction and cardiomyopathy on treatment with DOXO. On the same line, EPI is an analogue of DOXO that has an activity against many forms of cancer. EPI is believed to act by intercalating between DNA base pairs and uncoiling the DNA helix, which results in inhibition of DNA synthesis. EPI has proved to be active on a wide spectrum of experimental tumours including leukaemias and sarcomas. Both DOXO and EPI may significantly upregulate the expression of death receptors at both protein and mRNA levels. DOXO has been found to be more cardiotoxic than EPI. Future studies are recommended in order to check their dose regimes and associated cardiotoxicity.

\section{Acknowledgements}

Authors thank head and staff of Department of Zoology, Khalsa College Amritsar for their constant support throughout the work.

\section{Funding}

There is no funding source for the present study.

\section{Competing interest}

None declared.

\section{References}

1. Rizvi SFA, Tariq S, Mehdi M (2018) Anthracyclines: mechanism of action, classification, pharmacokinetics and future-A mini review. Int $J$ of Biotech \& Bioeng 4: 81-85. [Crossref]

2. Nikerel HT, Karabekmez MK, Klrdar B (2018) Doxorubicin induces an extensive transcriptional and metabolic rewiring in yeast cells. Sci Rep 8: 13672. [Crossref]

3. http://chemocare.com/chemotherapy/drug-info/doxorubicin.aspx

4. https://voice.ons.org/news-and-views/outpatient-oncology-drug-series-doxorubicin-isthe-infamous-red-devil

5. Chatterjee K, Zhang J, Honbo N, Karliner JS (2010) Doxorubicin cardiomyopathy Cardiology 115: 155-162

6. Ichikawa Y, Ghanefar M, Bayeva M, Wu R, Khechaduri A, et al. (2014) Cardiotoxicity of doxorubicin is mediated through mitochondrial iron accumulation. J Clin Invest 124: 617-630. [Crossref]

7. Koleini N, Kardami E (2017) Autophagy and mitophagy in the context of doxorubicininduced cardiotoxicity. Oncotarget 8: 46663-46680. [Crossref]

8. An L, Hu XW, Zhang S, Hu X, Song Z, et al. (2017) UVRAG Deficiency Exacerbates Doxorubicin-Induced Cardiotoxicity. Sci Rep 7: 43251. [Crossref]

9. Zhao L, Zhang B (2017) Doxorubicin induces cardiotoxicity through upregulation of death receptors mediated apoptosis in cardiomyocytes. Sci Rep 7: 44735. [Crossref]

10. Li DL, Wang ZV, Ding G, Tan W, Luo X, et al. (2016) Doxorubicin Blocks Cardiomyocyte Autophagic Flux by Inhibiting Lysosome Acidification. Circulation 133: 1668-1687. [Crossref]

11. Plosker GL, Faulds D (1993) Epirubicin A Review of its Pharmacodynamic and Pharmacokinetic Properties, and Therapeutic Use in Cancer Chemotherapy. Drugs 45: 788-856. [Crossref]

12. http://www.mhra.gov.uk/home/groups/par/documents/websiteresources/con2023491 pdf

13. https://livertox.nih.gov/DoxorubicinEpirubicinIdarubicin.html

14. Mouridsen HT, Alfthan C, Bastholt L, Bergh J, Dalmark M, et al. (2009) Current Status of Epirubicin (Farmorubicin) in the Treatment of Solid Tumours. Acta Oncologica 29: 257-285. [Crossref]

15. Vulsteke C, Pfeil AM, Maggen C, Schwenkglenks M, Pettengell R (2015) Clinical and genetic risk factors for epirubicin-induced cardiac toxicity in early breast cancer patients. Breast Cancer Res Treat 152: 67-76. [Crossref]

16. Feng YY, Yang ZJ (2015) Clinical Application of the Heart Rate Deceleration Capacity Test to Predict Epirubicin-induced Cardiotoxicity. J Cardiovasc Pharmacol 66: 371375. [Crossref]

17. Luo R, Cui H, Huang D, Li G (2017) Early assessment of the left ventricular function by epirubicin-induced cardiotoxicity in postoperative breast cancer patients. Echocardiography 34: 1601-1609. [Crossref]

18. Framarino-dei-Malatesta M, Perrone G, Giancotti A, Ventriglia F, Derme M (2015) A new entry in the list of fetal cardiotoxic drugs? Intrauterine death of one fetus in a twin pregnancy. Case report and review of literature. BMC Cancer 15: 951. [Crossref]

19. Khasraw M, Bell R, Dang C (2012) Epirubicin: is it like doxorubicin in breast cancer? A clinical review. Breast 21: 142-149. [Crossref]

20. Bi X, Deng Y, Zeng F, Zhu Y, Wu Y, Zhao C, et al (2009) Evaluation of epirubicininduced cardiotoxicity by two-dimensional strain echocardiography in breast cancer patients. J Huazhong Univ Sci Technolog Med Sci 29: 391-394. [Crossref]

21. Okura Y, Kawasaki T, Kanbayashi C, Sato N (2012) A case of epirubicinassociated cardiotoxicity progressing to life-threatening heart failure and splenic thromboembolism. Intern Med 51:1355-1360. [Crossref]

22. Stohr W, Paulides M, Brecht I, Kremers A, Treuner J, et al. (2006) Comparison of epirubicin and doxorubicin cardiotoxicity in children and adolescents treated within the German Cooperative Soft Tissue Sarcoma Study (CWS). J Cancer Res Clin Oncol 132: 35-40. [Crossref] 
23. Nistico C, Bria E, Cuppone F, Carpino A, Ferretti G, et al. (2007) Troponin-T and myoglobin plus echocardiographic evaluation for monitoring early cardiotoxicity of weekly epirubicin-paclitaxel in metastatic breast cancer patients. Anticancer Drugs 18: 227-232. [Crossref]
24. Yang F, Lei Q, Li L, He JC, Zeng J, et al. (2017) Delivery of epirubicin via slow infusion as a strategy to mitigate chemotherapy-induced cardiotoxicity. PLoS One 12: e0188025. [Crossref]

Copyright: (C2019 Singh Z. This is an open-access article distributed under the terms of the Creative Commons Attribution License, which permits unrestricted use, distribution, and reproduction in any medium, provided the original author and source are credited. 